07

\title{
Переключение бистабильных магнитных состояний сплава (NdSmDy)(FeCo)B вблизи спин-переориентационного перехода
}

\author{
() А.И. Дмитриев ${ }^{1}$, С.А. Костюченко ${ }^{2}$ \\ ${ }^{1}$ Институт проблем химической физики РАН, Черноголовка \\ ${ }^{2}$ Московский государственный университет им. М.В. Ломоносова \\ E-mail: aid@icp.ac.ru
}

Поступило в Редакцию 7 марта 2017 г.

Установлено, что в окрестности спин-переориентационного перехода в сплаве $(\mathrm{NdSmDy})(\mathrm{FeCo}) \mathrm{B}$ формируются бистабильные магнитные состояния с двумя равновозможными ориентациями вектора намагниченности, соответствующими различной полярности постоянного магнита. Определено критического значение напряженности магнитного поля $\sim 1 \mathrm{kOe}$, в котором происходит переключение бистабильных магнитных состояний образца. Показано, что полярность спеченных магнитов семейства $\mathrm{Nd}_{2} \mathrm{Fe}_{14} \mathrm{~B}$ в окрестности спин-переориентационного перехода может быть стабилизирована небольшим внешним магнитным полем, что открывает новые возможности для применения таких магнитов в криомагнитных системах.

DOI: 10.21883/PJTF.2017.14.44828.16763

Постоянные магниты семейства $\mathrm{Nd}_{2} \mathrm{Fe}_{14} \mathrm{~B}$ применяются в различных технических устройствах, работающих при комнатной температуре. В настоящее время предпринимаются попытки использовать эти сплавы в системах, работающих при низких температурах [1]. Редкоземельные магниты $\mathrm{Nd}_{2} \mathrm{Fe}_{14} \mathrm{~B}$ охлаждают, чтобы достичь наибольших значений остаточной намагниченности и коэрцитивной силы. В окрестности температуры $T_{S R}=135 \mathrm{~K}$ в сплавах серии $\mathrm{Nd}_{2} \mathrm{Fe}_{14}$ В происходит спонтанное изменение направления вектора намагниченности (изменение полярности магнита), вызванное спин-переориентационным переходом (СПП), что затрудняет их низкотемпературную эксплуатацию [2,3]. СПП в монокристаллических образцах хорошо изучен, поэтому расчет вели- 
чины намагниченности и ее направления, хотя и оперирует большим количеством варьируемых независимых параметров (шесть параметров кристаллического поля и один параметр межподрешеточного обменного взаимодействия [4]), все же становится возможным. Однако изготавливаемые промышленностью магниты представляют собой поликристаллы. В этом случае СПП зависит не только от состава сплава, но и от его микроструктуры. Существует очень мало работ, описывающих СПП в поликристаллических магнитах $[5,6]$. Численных моделей, описывающих пространственное распределение намагниченности поликристаллических образцов в окрестности СПП, не существует вовсе. Цель данной статьи состояла в установлении закономерностей СПП в поликристаллических образцах $(\mathrm{NdSmDy})(\mathrm{FeCo}) \mathrm{B}$, а также в поиске влияния магнитного поля на динамику переключения магнитных состояний сплава вблизи СПП.

Образцы $\left(\mathrm{Nd}_{0.62} \mathrm{Dy}_{0.33} \mathrm{Sm}_{0.05}\right)_{16.2}\left(\mathrm{Fe}_{0.77} \mathrm{Co}_{0.23}\right)_{78.1} \mathrm{~B}_{5.7}$ были получены методом прессования и спекания порошкового сплава. Подробно методики приготовления и аттестации образцов приведены в работах $[3,7]$. Температурные зависимости намагниченности были измерены с помощью СКВИД-магнитометра MPMS 5XL Quantum Design. На рис. 1, $a$ приведены температурные зависимости намагниченности $M(T)$ образца, измеренные в магнитных полях различной напряженности. Вблизи температуры $T_{S R}=110 \mathrm{~K}$ в образце происходит СПП, при котором в магнитных полях $H<150$ Ое намагниченность уменьшается (рис. 1,a). Напротив, в магнитных полях $H>250$ Ое намагниченность увеличивается (рис. $1, a)$.

Положим, что при температурах выше температуры СПП ось легкого намагничивания направлена вдоль оси $z$ (рис. $1, b)$. Энергия $E$ одноосного ферромагнитного образца в магнитном поле $H=\left(H_{x}, 0, H_{z}\right)$ описывается выражением [8]

$$
E=K_{1} \sin ^{2} \theta+K_{2} \sin ^{4} \theta-M_{s} H_{x} \sin \theta-M_{s} H_{z} \cos \theta,
$$

где первые два слагаемых соответствуют энергии магнитной анизотропии, вторые два слагаемых - магнитостатической энергии. Здесь $K_{1}-$ константа магнитной анизотропии первого порядка, $K_{2}-$ константа магнитной анизотропии второго порядка, $M_{s}$ - намагниченность насыщения, $\theta$ - угол между осью легкого намагничивания $z$ и вектором намагниченности (рис. $1, b)$. Минимизация полной энергии $\partial E / \partial \theta=0$ 

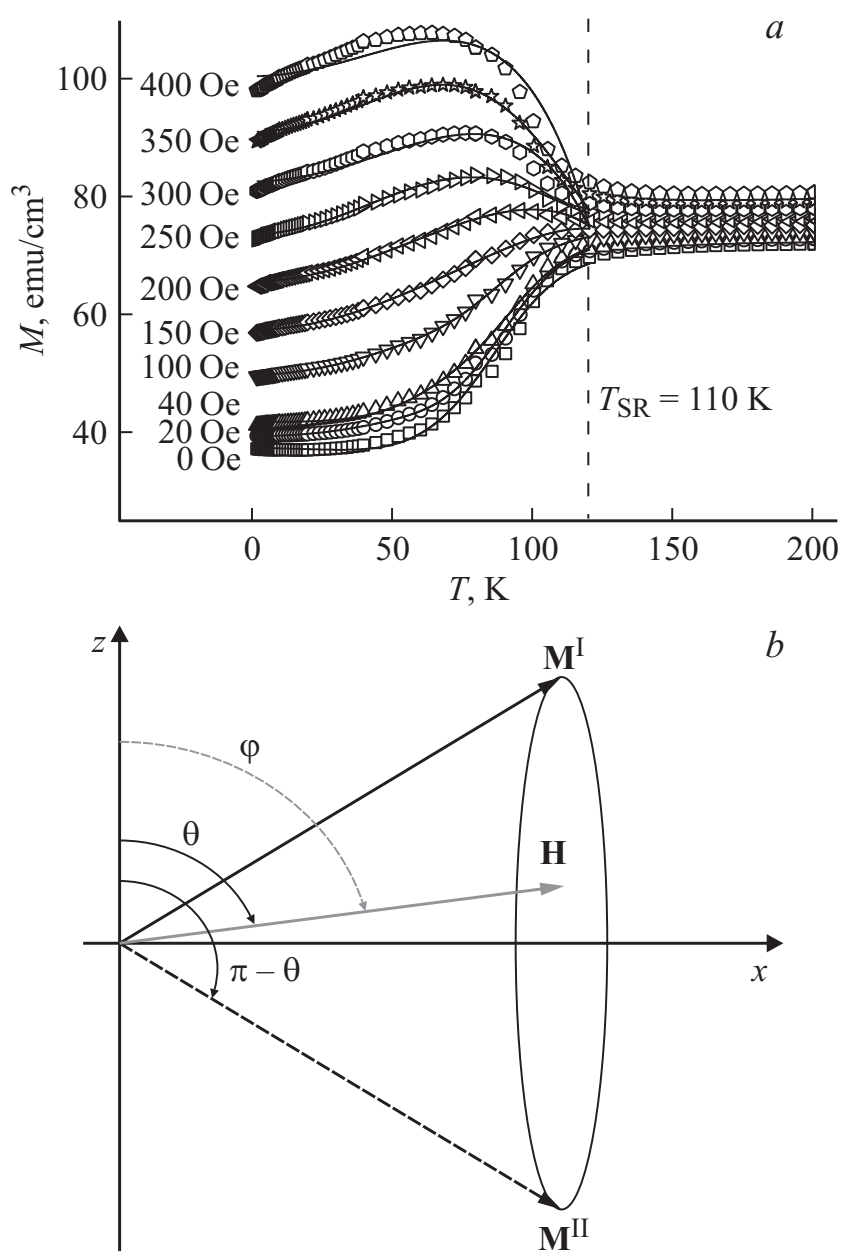

Рис. 1. $a-$ температурные зависимости намагниченности образца; $b-$ схема ориентации вектора намагниченности относительно осей $z$ и $x$.

приводит к алгебраическому уравнению четвертой степени относительно неизвестной $\sin \theta$. В магнитном поле $H=H_{x}$, приложенном перпендикулярно легкой оси $z$, кривая намагничивания одноосного

Письма в ЖТФ, 2017, том 43, вып. 14 
ферромагнетика описывается выражением [8]

$$
M_{x}=M_{s} \sqrt{\frac{2\left|K_{1}\right|}{3 K_{2}}} \cos \left(\frac{1}{3} \arccos \frac{3 M_{s} \sqrt{\frac{6 K_{2}}{\left|K_{1}\right|}}}{4\left|K_{1}\right|} H_{x}\right) .
$$

В магнитном поле $H=H_{z}$, приложенном вдоль легкой оси $z$, кривая намагничивания описывается выражением [8]

$$
M_{z}=2 M_{s} \sqrt{\frac{\frac{2 K_{2}}{|K|_{1}}-1}{\frac{6 K_{2}}{\left|K_{1}\right|}}} \cos \left(\frac{1}{3} \arccos \frac{3 M_{s} \sqrt{\frac{6 K_{2}}{\left|K_{1}\right|}}}{4\left|K_{1}\right|\left(\frac{2 K_{2}}{\left|K_{1}\right|}-1\right)^{3 / 2}} H_{z}\right) .
$$

В работе [6] было показано, что температурные зависимости констант магнитной анизотропии сплава $(\mathrm{NdSmDy})(\mathrm{FeCo}) \mathrm{B}$ описываются формулой Брюхатова-Киренского

$$
K_{i}=K_{0_{i}} \exp \left(-a_{i} T^{2}\right)+b_{i},
$$

где значение $i=1$ соответствует $K_{1}$, значение $i=2$ соответствует $K_{2}$. Здесь $K_{0}, a, b-$ феноменологические константы. На рис. 2 приведены расчетные температурные зависимости согласно уравнениям (2)-(4) обеих проекций намагниченности, нормированных на $M_{s}$. Используемые при расчете значения констант $K_{0_{i}}, a_{i}, b_{i}$ взяты из работы [6]. Полученные расчетные зависимости $M_{x}$ и $M_{z}$ идентичны экспериментальным зависимостям намагниченности монокристалла $\mathrm{Nd}_{2} \mathrm{Fe}_{14} \mathrm{~B}$, измеренным в работе [3]. Когда магнитное поле Н приложено перпендикулярно к оси легкого намагничивания $\left(H_{x}=H, H_{z}=0\right)$, возникают особые бистабильные магнитные состояния с двумя равновозможными ориентациями вектора намагниченности М с углами $\theta$ и $\pi-\theta[9]$ (рис. $1, b$ ). В поликристаллическом образце часть зерен разориентирована. Это означает, что для них имеется ненулевая проекция $H_{z}$, что эквивалентно монокристаллу, повернутому относительно магнитного поля $\mathbf{H}$ на некоторый угол $\varphi$. Угол $\varphi$ можно интерпретировать как эффективную меру разориентации отдельных зерен в поликристаллическом образце. В этом случае измеряемая проекция намагниченности на направление магнитного поля складывается из обеих проекций $M_{x}$ и $M_{z}$.

Письма в ЖТФ, 2017, том 43, вып. 14 


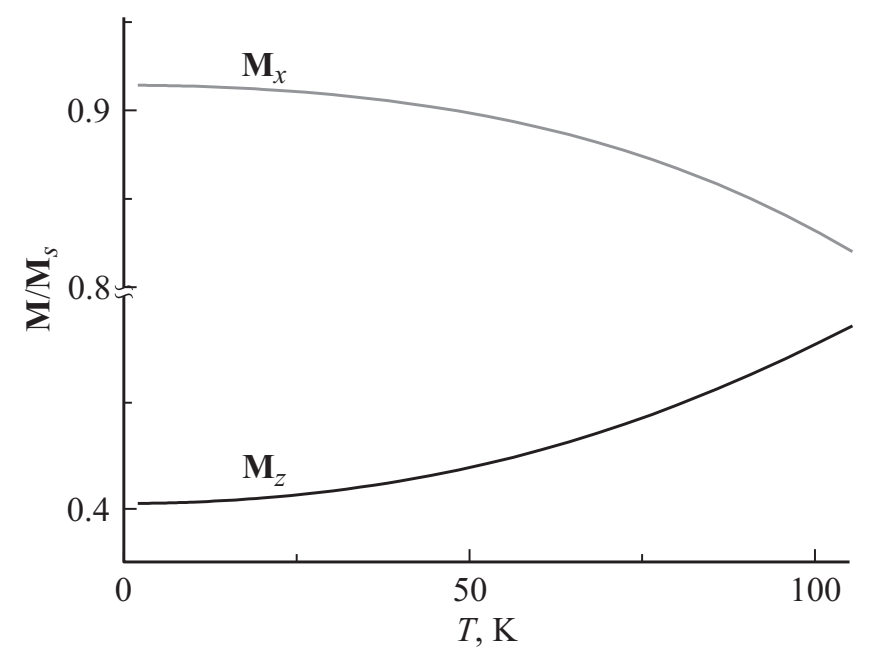

Рис. 2. Расчетные температурные зависимости проекций намагниченности $M_{x}$ (серая кривая) и $M_{z}$ (черная кривая).

Легко показать, что одно из рассмотренных выше магнитных состояний, соответствующее углу $\theta$, характеризуется высокой намагниченностью $M^{\mathrm{I}}=M_{x} \sin \varphi+M_{z} \cos \varphi$. Оно остается термодинамически вполне устойчиво. Второе магнитное состояние, соответствующее углу $\pi-\theta$, характеризуется низкой намагниченностью $M^{\mathrm{II}}=M_{x} \sin \varphi-M_{z} \cos \varphi$. Оно становится термодинамически метастабильно. В общем случае в поликристаллическом образце имеет место сосуществование обоих состояний, а кривая намагничивания является суперпозицией величин $M^{\mathrm{I}}$ и $M^{\mathrm{II}}$ :

$$
M=\frac{p M^{\mathrm{I}}+q M^{\mathrm{II}}}{p+q},
$$

где $p$ и $q-$ доли рассмотренных выше магнитных фаз $M^{\mathrm{I}}$ и $M^{\mathrm{II}}$ соответственно. При наложении магнитного поля происходит переключение этих состояний. На рис. 1, $a$ сплошными линиями показаны аппроксимации экспериментальных температурных зависимостей намагниченности выражением (5). Из аппроксимации были определены значения $p$ для каждого значения напряженности магнитного поля.

Письма в ЖТФ, 2017, том 43, вып. 14 


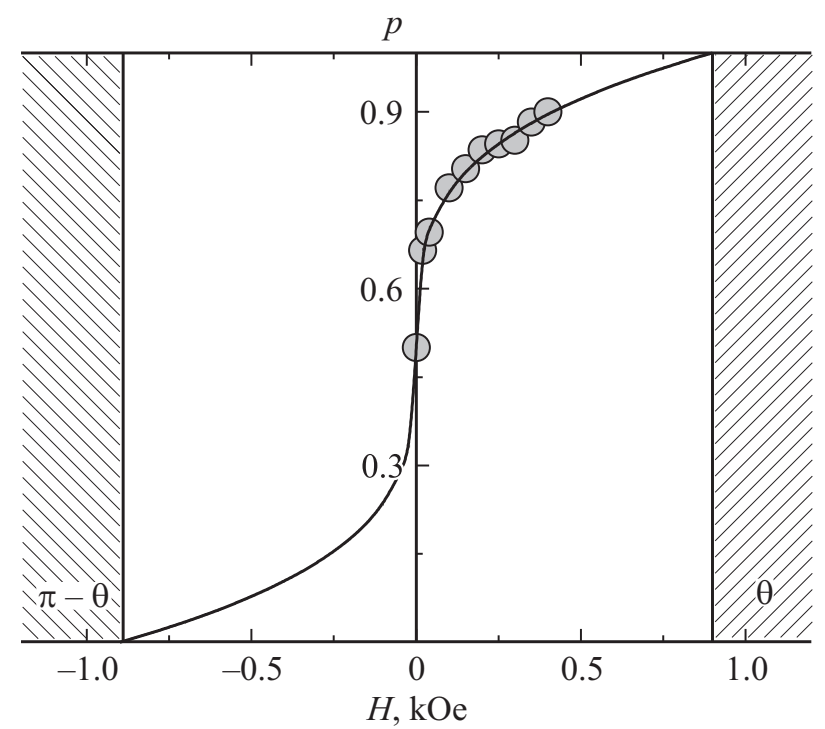

Рис. 3. Фазовая $(p, H)$-диаграмма образца.

Зависимость $p(H)$ приведена на рис. 3. Сплошной линией показана аппроксимация зависимости $p(H)$ выражением $p_{0}+\left(H / H_{0}\right)^{1 / 3}$. Здесь $p_{0}=0.5$ - значение функции $p(H)$ в нулевом магнитном поле, соответствующее фазовому равновесию; $H_{0}=1 \mathrm{kOe}-$ критическое значение напряженности магнитного поля, в котором происходит переключение бистабильных магнитных состояний образца. Рис. 3 является фазовой диаграммой в координатах $(p, H)$, на которой сплошная линия есть линия фазового перехода из состояния с ориентацией вектора М под углом $\pi-\theta$ в магнитных полях $H<-1 \mathrm{kOe}$ в состояние с ориентацией вектора М под углом $\theta$ в магнитных полях $H>1 \mathrm{kOe}$.

Таким образом, установлено, что в окрестности СПП в магнитных полях напряженностью $H<150$ Ое намагниченность сплава ( $\mathrm{NdSmDy}$ (FeCo)B уменьшается при понижении температуры, напротив, в магнитных полях напряженностью $H>250$ Ое намагниченность образца увеличивается при понижении температуры. Такое поведение зависимостей $M(T)$ обусловлено вкладом в суммарную намагниченность обеих проекций $M_{x}$ и $M_{z}$. Определено критическое значение 
напряженности магнитного поля $H_{0}=1 \mathrm{kOe}$, в котором происходит переключение бистабильных магнитных состояний образца с двумя равновозможными ориентациями намагниченности. Полярность поликристаллических спеченных магнитов семейства $\mathrm{Nd}_{2} \mathrm{Fe}_{14} \mathrm{~B}$ в окрестности спин-переориентационного перехода может быть стабилизирована с помощью внешнего подмагничивающего поля, что открывает возможности для их применения в криомагнитных системах.

Работа поддержана грантом РФФИ № 16-07-00863_а. Авторы признательны Р.Б. Моргунову за предоставленные образцы.

\section{Список литературы}

[1] Hara T., Tanaka T., Kitamura H. et al. // Phys. Rev. ST Accel. Beams. 2004. V. 7. P. 050702.

[2] Hirosawa S., Matsuura Y., Yamamoto H. et al. // J. Appl. Phys. 1986. V. 59. P. 873-879.

[3] Koon N.C., Das B.N., Rubinstein M. et al. // J. Appl. Phys. 1985. V. 57. P. 40914093.

[4] Мушников Н.В., Терентьев П.Б., Розенфельд Е.В. // ФММ. 2007. Т. 103. C. $42-53$.

[5] Pinkerton F.E. // J. Appl. Phys. 1988. V. 64. P. 5565-5567.

[6] Дмитриев А.И. // ФТТ. 2016. Т. 58. С. 2361-2364.

[7] Kablov E.N., Ospennikova O.G., Kablov D.E. et al. // J. Appl. Phys. 2015. V. 117. P. 243903.

[8] Мищек А.И., Колмакова Н.П., Сирота Д.И. // ФММ. 1974. Т. 38. С. 35-47.

[9] Ландау Л.Д., Лифиии, E.M. Теоретическая физика. Электродинамика сплошных сред. М.: Наука, 1982. 624 с.

Письма в ЖТФ, 2017, том 43, вып. 14 